\title{
Objective quantification of perturbations produced with a piecewise PV inversion technique
}

\author{
L. Fita, R. Romero, and C. Ramis \\ Grup de Meteorologia, Departament de Física, Universitat de les Illes Balears, Ciutat de Mallorca, Spain \\ Received: 14 May 2007 - Revised: 21 September 2007 - Accepted: 23 October 2007 - Published: 29 November 2007
}

\begin{abstract}
PV inversion techniques have been widely used in numerical studies of severe weather cases. These techniques can be applied as a way to study the sensitivity of the responsible meteorological system to changes in the initial conditions of the simulations. Dynamical effects of a collection of atmospheric features involved in the evolution of the system can be isolated. However, aspects, such as the definition of the atmospheric features or the amount of change in the initial conditions, are largely case-dependent and/or subjectively defined. An objective way to calculate the modification of the initial fields is proposed to alleviate this problem. The perturbations are quantified as the mean absolute variations of the total energy between the original and modified fields, and an unique energy variation value is fixed for all the perturbations derived from different PV anomalies. Thus, PV features of different dimensions and characteristics introduce the same net modification of the initial conditions from an energetic point of view. The devised quantification method is applied to study the high impact weather case of 9-11 November 2001 in the Western Mediterranean basin, when a deep and strong cyclone was formed. On the Balearic Islands 4 people died, and sustained winds of $30 \mathrm{~ms}^{-1}$ and precipitation higher than $200 \mathrm{~mm} / 24 \mathrm{~h}$ were recorded. Moreover, 700 people died in Algiers during the first phase of the event. The sensitivities to perturbations in the initial conditions of a deep upper level trough, the anticyclonic system related to the North Atlantic high and the surface thermal anomaly related to the baroclinicity of the environment are determined. Results reveal a high influence of the upper level trough and the surface thermal anomaly and a minor role of the North Atlantic high during the genesis of the cyclone.
\end{abstract}

Keywords. Meteorology and atmospheric dynamics (Mesoscale meteorology; Middle atmosphere dynamics)

Correspondence to: L. Fita

(lluis.fita@uib.es)

\section{Introduction}

Potential Vorticity (PV) as a conservative quantity for adiabatic and frictionless conditions has been revealed as a good variable to study the structure and evolution of cyclones (Hoskins et al., 1985; Gyakum, 1983b; Huo et al., 1995; Hakim et al., 1996). The piecewise PV inversion technique (Davis and Emanuel, 1991) can be used as a way to modify the initial conditions of numerical simulations. The sensitivity to changes in the initial conditions has been used in several dynamical studies of intense storms (Huo et al., 1999a,b; Romero, 2001a; Homar et al., 2002, 2003). This approach has been shown as a powerful tool towards the understanding of important atmospheric aspects related to the cyclogenesis, such as baroclinic and barotropic development and convection. Piecewise PV inversion allows one to study the impact of the selected PV anomalies on the structure of the initial atmospheric fields and on the subsequent dynamical evolution of the simulated circulation systems.

However, the method of designing perturbed simulations via PV inversion exhibits some particular aspects that are case-dependent, and presents some subjective choices that can deeply influence the results. Some of them are: identification of the PV features, magnitude of the modification of the initial conditions, a set of balance equations used in the inversion, and boundary conditions for the inversion. In this study, the piecewise PV inversion technique of Davis and Emanuel (1991) is used. In order to diminish the subjectivity of the procedure, an objective way to quantify the initial modification introduced in the perturbed simulations is proposed. The quantification of the perturbation is expressed as the mean absolute variation of the total energy between the initial and the modified fields, considering the whole horizontal domain of simulation and all vertical levels. The capability to compute the "total amount" of the introduced modification makes an objective control of one important aspect of the PV inversion technique possible, favouring meaningful intercomparisons of perturbed scenarios.

Published by Copernicus Publications on behalf of the European Geosciences Union. 

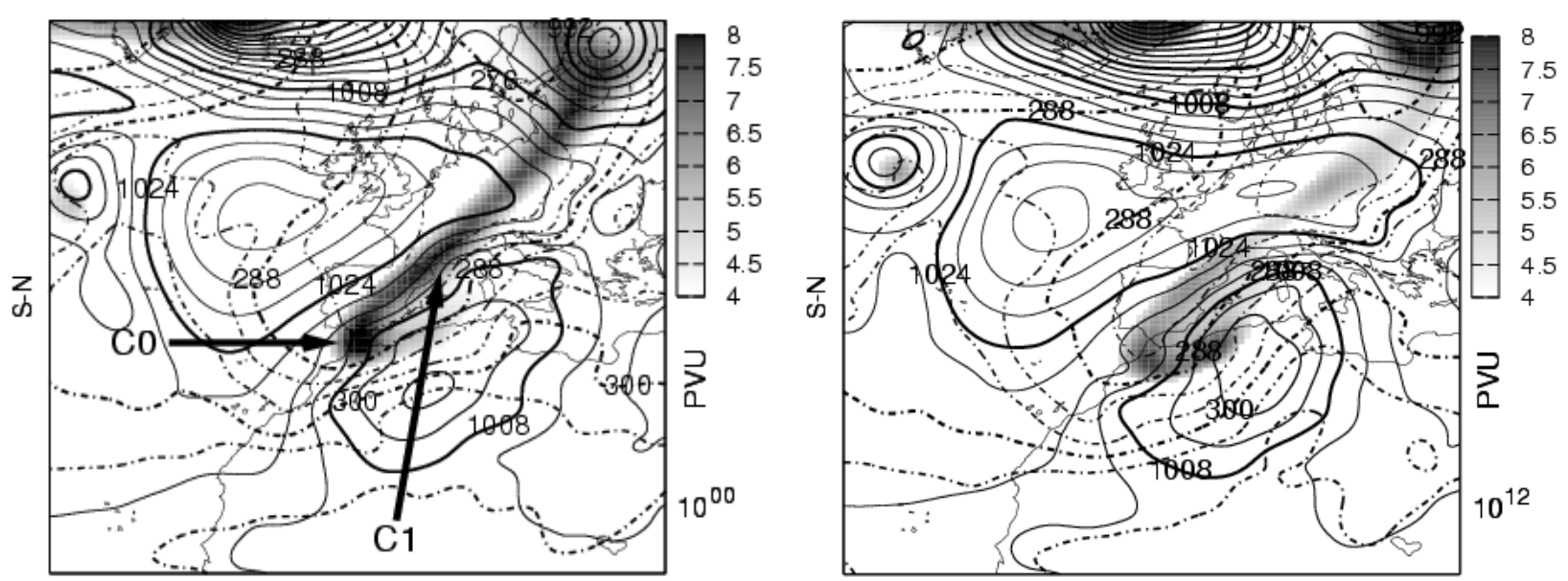

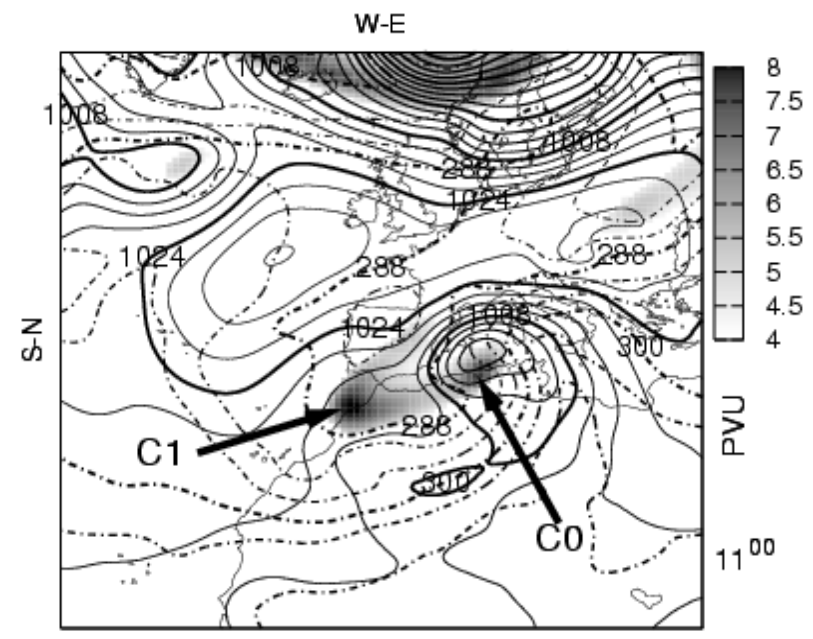

W-E

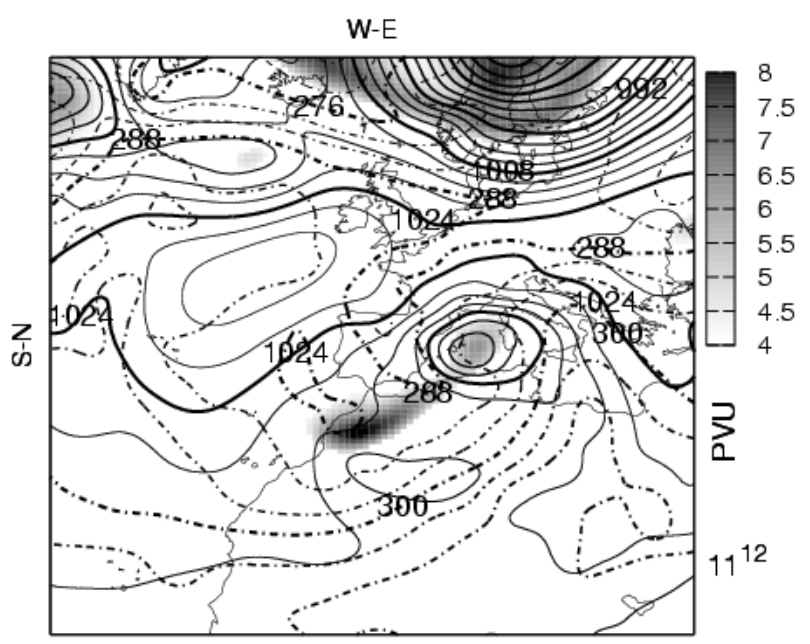

W-E

Fig. 1. Sea level pressure (every $4 \mathrm{hPa}$, solid line), Potential temperature at $850 \mathrm{hPa}$ (every $4 \mathrm{~K}$, dashed line) and Isentropic Ertel PV at $330 \mathrm{~K}\left(1 \mathrm{PVU}=10^{-6} \mathrm{~m}^{2} \mathrm{Ks}^{-1} \mathrm{~kg}^{-1}\right.$, coloured field) from ECMWF analyses on 10 November 2001 at 00:00 UTC (top left), 10 November at 12:00 UTC (top right), 11 November at 00:00 UTC (bottom left) and 11 November at 12:00 UTC (bottom right).

The 9-12 November 2001 cyclone has been described by the authors as one of the most intense events in the Western Mediterranean basin during the last 25 years. The case is a deep episode of a three-dimensional cyclone classification done by Campins et al. (2006). It has been also widely studied (Davolio and Buzzi, 2004; Tripoli et al., 2005; Argence et al., 2006). In $24 \mathrm{~h}$ a strong and deep vortex was formed. As a result, heavy precipitation and strong winds were recorded in Algiers and in the Balearic Islands. Seven hundred people died as a result of severe floods in Algiers, and 4 people died in the Balearic Islands, where sustained winds of $30 \mathrm{~ms}^{-1}$ and precipitation above $200 \mathrm{~mm} / 24 \mathrm{~h}$ were recorded and about two million trees fell down on the Mallorca Island.
The work is structured as follows: the second section describes the cyclone event, followed by a discussion and formulation of the energy-quantification method of the initial perturbed conditions (Sect. 3). A fourth section with the results of the November 2001 storm is presented, and a final section of conclusions is also given.

\section{Case description}

The case is a clear and strong example of a Pettersen-Smeybe class B cyclone evolution (Pettersen and Smebye, 1971), in which a cyclone is formed in a pre-existing upper level trough environment. The genesis and evolution of the case presents similar characteristics to the case that occurred in December 1979 in the Western Mediterranean basin (Homar 
et al., 2002). On 10 November 2001, a disturbance was formed at the surface to the south of the Atlas mountains range (see Fig. 1, with the ECMWF analyses maps). A significant upper level disturbance was located over central Europe, extending from the Iberian peninsula to the Baltic Sea. This upper level disturbance shows a structure of two high PV positive anomalies ( $\mathrm{C}, \mathrm{C} 1$, see labels in Fig. 1). Over the Mediterranean Sea there was a post storm situation with residual convective activity (not shown). As a result of the surface African low and the upper level disturbance, strong thermal gradients developed over the western Mediterranean basin. The low level cyclone moved northwards; meanwhile, the upper level disturbance translated southwards. While the low-level low crossed the Atlas mountains, the upper level PV positive centres described a singular relative rotational movement, attributed to their interactions through PV advection. Mutual interaction between different positive PV vortices has been shown as an important factor that contributes to the cyclogenesis (Hakim et al., 1996). Although in this case, there was not a merging of the PV centres.

On 10 November, at 18:00 UTC, a strong interaction between upper level and low level disturbances was established. This is identified in Fig. 2 as the closeness between the upper level disturbance (see the tropopause fold, or free cloud area), and a strong cyclonic cloud structure (cyclone, indicated by curved arrows). These configurations of proximity between strong surface thermal gradients and upper level trough disturbances become the ideal environmental conditions, under an appropriate vertical wind shear, to produce the baroclinic growth of disturbances. It has been described as one of the most important baroclinic processes from which deep cyclones can be formed (Hoskins et al., 1985; Bleck, 1990). From that time the cyclone crossed the Algerian coast and reached its mature state (11 November at 00:00 UTC), while strong cloud formation was present in the area. Strong winds were produced at the surface $\left(33 \mathrm{~ms}^{-1}\right.$ sustained wind records were registered on the Balearic Islands between 11 November 00:00 UTC and 06:00 UTC). Some time later the upper-low level interaction weakened because of the southward movement of the upper level disturbance and the northward motion of the cyclone. At this point the cyclone weakened and could be described as an eastward movement while it approached the Sardinia Island.

The case will be studied using the MM5 nonhydrostatic primitive equation mesoscale model (Grell et al., 1994). A control simulation is run with one domain, with a horizontal resolution of $54 \mathrm{~km}$ and 23 vertical levels. The simulated period comprises the interval between 10 November 2001 at 00:00 UTC and 12 November 2001 at 00:00 UTC. Control simulation will prescribe the main characteristics of the cyclone that will be compared to the simulated cyclones obtained from the PV-based sensitivity tests. All MM5 simulations will be run in the same configuration based on a graupel(reisner2) scheme for the explicit moisture processes, a Kain-Fritsch scheme for the cumulus convection, a MRF

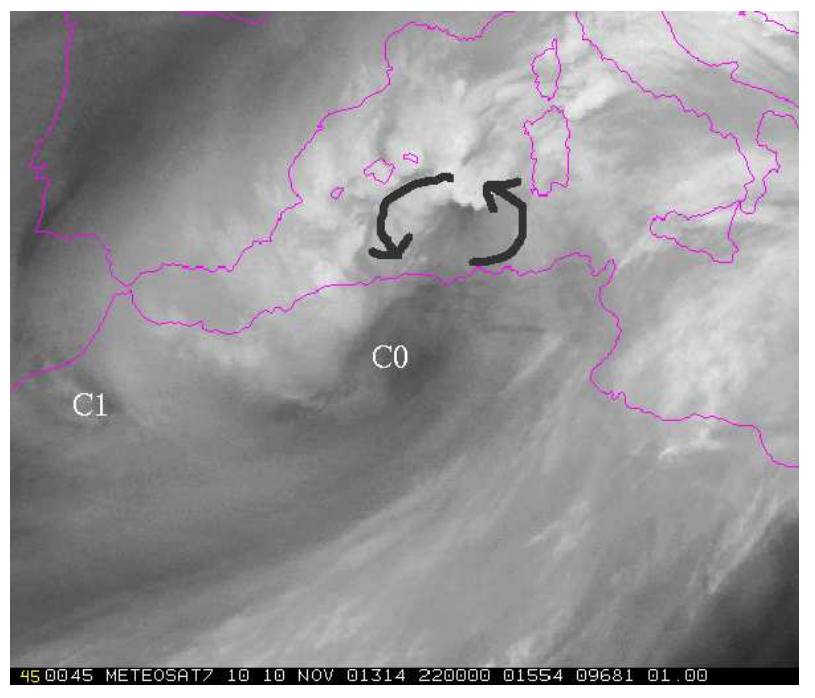

Fig. 2. Normalised Water Vapour METEOSAT7 image on 10 November 2001 at 22:00 UTC. EUMETSAT source.

parametrisation for the planetary boundary layer and the "rrtm" scheme (long wave) for the atmospheric radiation.

In order to track the baroclinic mechanism, the trajectories of "C0" (the southwestern upper level PV vortex inside the upper level trough on 10 November at 00:00 UTC, Fig. 1 of ECMWF analyses) and the surface cyclone are analysed. During the initial phase of the evolution, control simulation results show that meanwhile, the upper level positive vortex $\mathrm{C} 0$ has moved southward, the cyclone has moved northward. In this way the low-level disturbance and $\mathrm{C} 0$ became closer (10 November at 12:00 UTC in Fig. 3). While the relative horizontal distance between the upper and low -level disturbances decreased, the cyclone and $\mathrm{C} 0$ increased their intensity as a reflection of the baroclinic theory of phase coupling between the upper and low level disturbances (Hoskins et al., 1985). However, the cyclone reached its mature state on a negative baroclinic-phase ( $\mathrm{C} 0$ is located east relative to the cyclone, Fig. 3 on 11 November at 00:00 UTC).

In this phase of the cyclone evolution, the cyclone reached the Mediterranean coast. Latent Heat Flux from the sea surface and the vigorous release of latent heat due to a strong cloud formation at mid levels (see water vapour satellite image in Fig. 2) could contribute to the intensification of the cyclone. An intensification of cyclones, due to the diabatic effects induced by the sea, has been detected in other cases, like in the North Atlantic "bombs" (Sanders and Gyakum, 1980; Kuo et al., 1991a,b), or other Mediterranean cases (Homar et al., 2002; Romero, 2001a). Moreover, the block-phase mutual interaction of the baroclinic process is also shown by the increasing central value of the C0 (Fig. 4). A significantly different behaviour between the two upper level vortices is observed in the same figure; whereas $\mathrm{C} 0$ became deeper, the other centre $(\mathrm{C} 1)$ remained constant during the mutual 

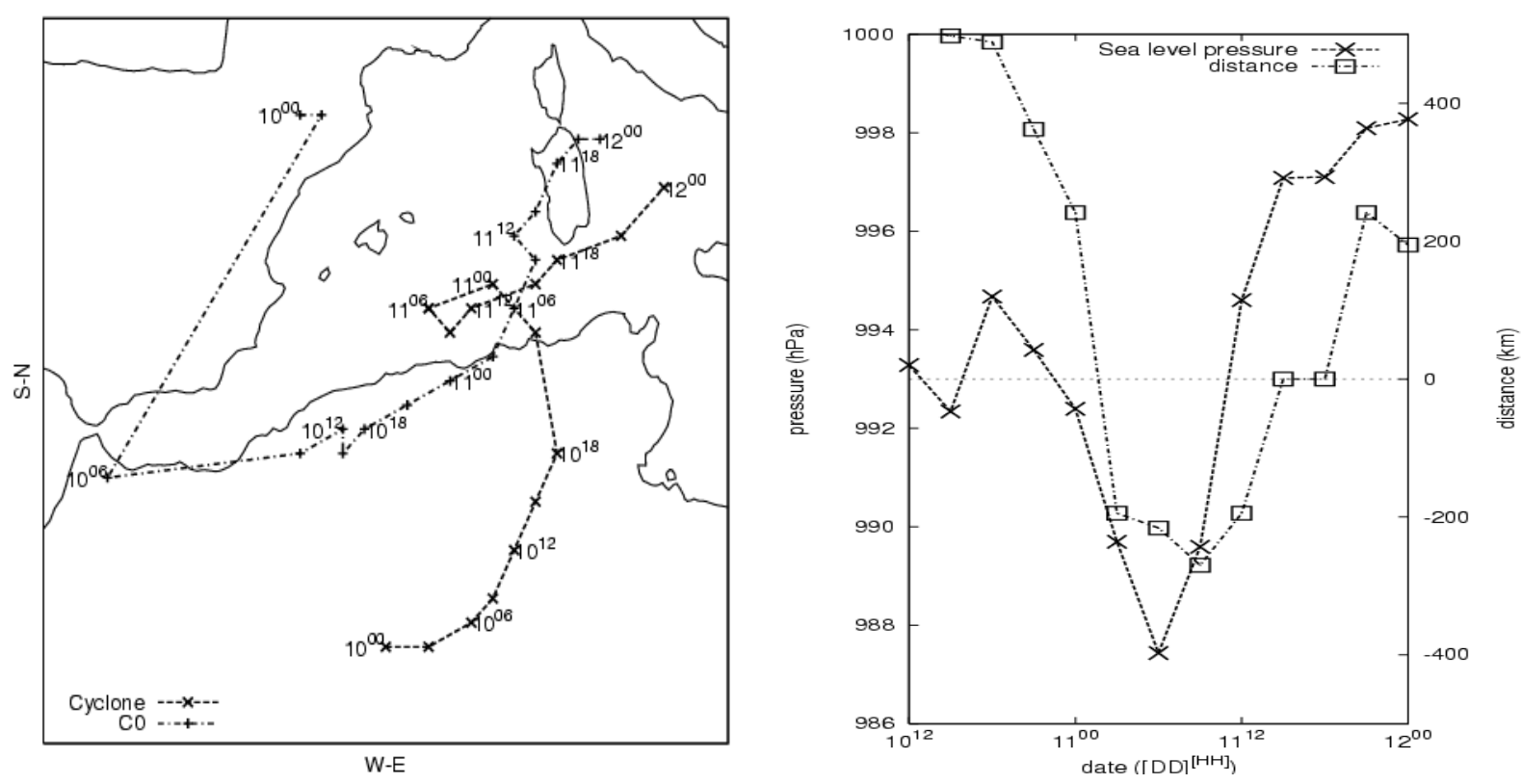

Fig. 3. Control simulation results. Left panel: Evolution of the position of the cyclone (dashed line) and the C0 (slash-dot line), date of position is included $\left([\mathrm{DD}]^{[\mathrm{HH}]}\right)$. Right panel: Evolution of the central pressure of the cyclone (hPa, y-axis left) and its relative horizontal distance with $\mathrm{C} 0$ (km, y-axis right) since 10 November at 12:00 UTC. Relative horizontal distance is the distance between the positions of the centre of $\mathrm{C} 0$ and the cyclone. Positive(Negative) values of the relative distance occur when the cyclone is located eastward (westward) from $\mathrm{C} 0$.

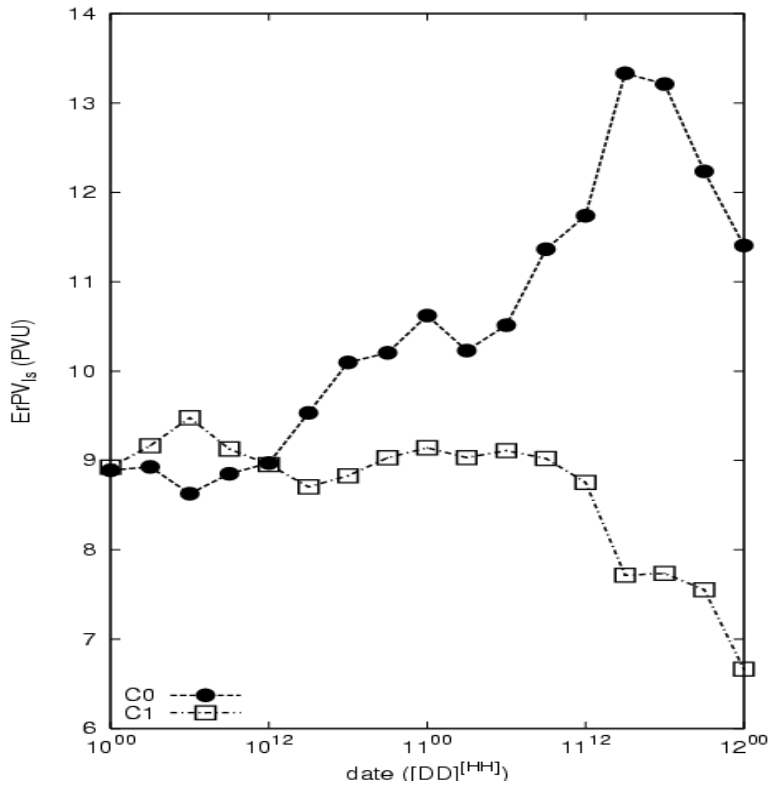

Fig. 4. Simulated central value (PVU) of the two upper level vortices $\mathrm{C} 0$ and $\mathrm{C} 1$, that are embedded within the upper level trough.

interaction period (10 November 15:00 UTC to 11 November 00:00 UTC; see Fig. 4). It is shown how $\mathrm{C} 0$ preserves its PV during the African phase of the cyclone. However, when the cyclone reached the sea, strong diabatic processes developed and $\mathrm{C} 0$ suffered an important increasing of its PV value. Meanwhile, $\mathrm{C} 1$ conserves its PV during almost the entire period of simulation.

During the African phase of the cyclone evolution (from 10 November 00:00 UTC to 10 November 18:00 UTC), the cyclone shows low vorticity (see Fig. 5). When the cyclone reached the sea it attained the mature state (11 November 00:00 UTC), depicted as: lowest surface central pressure value $(988 \mathrm{hPa})$ and strong geostrophic vorticity (more than $30 \times 10^{-5} \mathrm{~s}^{-1}$ at the centre of the cyclone, computed using $200 \mathrm{~km}$ resolution geopotential data as in Campins et al., 2000).

\section{Objective quantification of perturbations}

The piecewise PV Inversion technique explained in Davis and Emanuel (1991) is used in the study. It is implemented over the $100 \times 120(54 \mathrm{~km}$ resolution $)$ horizontally discretized domain and 21 isobaric vertical levels, from $1000 \mathrm{hPa}$ to $100 \mathrm{hPa}$. Ertel PV field is inverted using a Charney equation of balance (Charney, 1955) with Dirichlet and Neumann boundary conditions at lateral and vertical boundaries (see reference for more details). Due to the linearization of the method, individual pieces of the Ertel PV field can be inverted individually, allowing for the study of different 


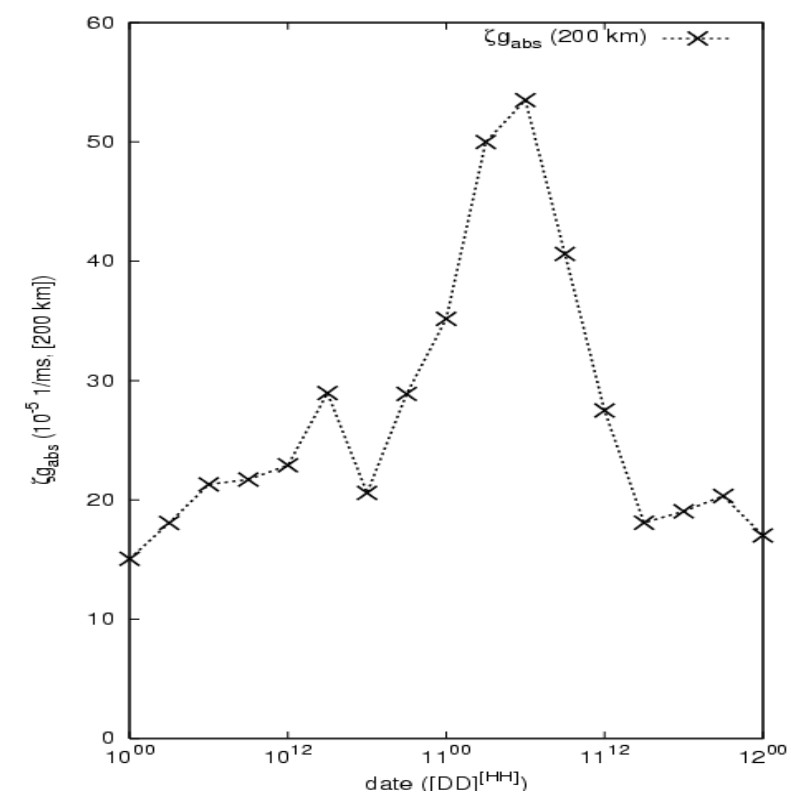

Fig. 5. Evolution of the geostrophic vorticity calculated at $200 \mathrm{~km}$ (defined as in Campins et al., 2000), using a constant Coriolis value of $C_{0}=8.25 \times 10^{-6} \mathrm{~s}^{-1}$, and density $\rho=1.225 \mathrm{~kg} \mathrm{~m}^{-3}$.

features of the environment to which they are related. These individual components of the PV field will be identified with relevant anomalies from a reference state of the atmosphere, and then inverted to produce the initial perturbations in the simulations. As a reference state, a seven-day average of the ECMWF analyses fields is computed (from 8 November 2001 at 00:00 UTC to 14 November at 00:00 UTC). Ertel PV anomalies are defined as the difference between the Ertel PV field on 10 November at 00:00 UTC (simulation start time) and the 7-day time-average ErPV. In this study the role of three major anomalies is analysed: (1) the upper level positive PV anomaly related to the two main upper level disturbances, (2) upper level negative PV anomaly related to the North Atlantic high and (3) the surface thermal anomaly related to the initial baroclinicity of the environment. Theoretically, a positive(negative) surface thermal anomaly can be related to a formal positive(negative) PV anomaly located below the bottom boundary (Bretherton, 1966; Thorpe, 1986; Reed et al., 1992; Horvath et al., 2006). In practice, the surface thermal anomaly is introduced as a bottom boundary field in the PV inversion method. Inthis way, the inverted balanced fields will be equivalent to the inverted fields that one can obtain from a PV anomaly under the ground (surrogate PV) that would produce the same surface thermal anomaly.

The satellite image (Fig. 2) depicts that deep convection developed. In other studies, like Davis and Emanuel (1991); Huo et al. (1999a); Reed et al. (1992), cloudy systems are also treated as PV anomalies. During cloud formation, there is a strong release of heat at mid levels due to water vapour
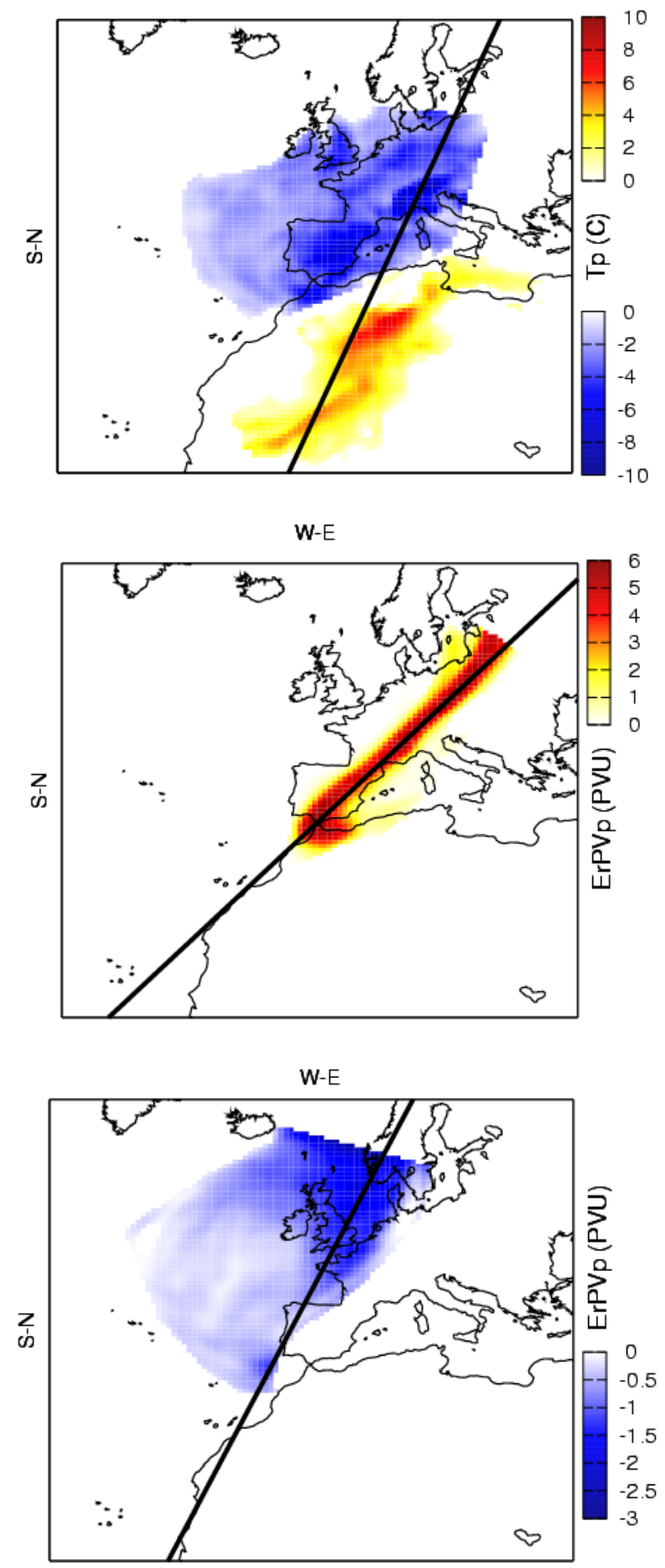

W-E

Fig. 6. Surface thermal anomaly defined as ErPVpTerm (top; yellow-red, positive values; white-blue, negative values), Upper level PV perturbation $(300 \mathrm{hPa})$ related to upper level trough defined as ErPVp01 (middle), Upper level PV perturbation $(300 \mathrm{hPa})$ related to North Atlantic High defined as ErPVpHigh (bottom), different scales are used. Solid lines show different vertical cross sections. 
Table 1. Percentage of modifications of the Newtoninan-obtained inverted fields of each anomaly (\%) used to modify the initial conditions. Mean absolute variation of the total energy $(\mathcal{M A \mathcal { V }})$ introduced on the initial conditions $(+$, increasing case; -, decreasing case).

\begin{tabular}{cccccccc}
\hline \multirow{2}{*}{ Final values } & \multicolumn{2}{c}{ ErPVpTerm } & \multicolumn{2}{c}{ ErPVp01 } & \multicolumn{2}{c}{ ErPVpHigh } \\
& & $\%$ & $\delta_{E N G}$ & $\%$ & $\delta_{E N G}$ & $\%$ & $\delta_{E N G}$ \\
\hline+ & $\mathcal{M A V}$ & 70 & 0.14504 & 8.8780 & 0.14504 & 7.6002 & 0.14505 \\
- & $\mathcal{M} \mathcal{A V}$ & 70 & 0.14508 & 8.8002 & 0.14509 & 7.6401 & 0.14508 \\
\hline
\end{tabular}

condensation. The increase in temperature at mid levels enhances the stability beneath, which is reflected as positive PV generation (Hoskins et al., 1985; Davis and Emanuel, 1991). However, low-level $(p>500 \mathrm{hPa})$ and moist $(\mathrm{RH}>70 \%)$ initial PV anomalies did not play an important role in preliminar simulations (not shown). This might be due to the fact that the cyclone was formed above the dry and hot Saharan desert and a deep convection was developed when the cyclone reached the sea $(18 \mathrm{~h}$ later than the time when the modification of initial conditions was done).

Selected anomalies are morphologically and spatially different (see Fig. 6). The modification of the model initial conditions derived from these might be significantly different. In order to compare the roles of the PV anomalies, a "normalisation" of the perturbation should be done. Then one could be sure that the total amount of change introduced by means of the inverted fields remains constant among the simulations initially, independent of the size and strength of the anomaly. Thus, the results can be adequately intercompared: the nonlinear evolution of the atmosphere will show the sensitivity of the event to changes in the initial conditions of the simulations. The

PV Inversion technique applied at each PV anomaly produces three-dimensional balanced fields (geopotential, temperature, stream function/horizontal wind). The inversion of the equations is numerically done through the overrelaxation method. For more details, refer to Davis and Emanuel (1991). The modification of the initial conditions will be done by a substraction of a percentage of these inverted fields. Since the inverted fields are obtained for the entire space, the normalisation or quantification of the perturbation requires a three-dimensional variable, and since PV inverted fields represent all atmospheric variables (except humidity), the index used for the normalisation should be a combination of these variables. The total energy (Bluestein, 1992) has been chosen as a plausible function. Using the energy, an energy-derived dynamical study of each anomaly can be accomplished. This is possible since total energy can be split into three components: kinetic $\left(\delta \mathcal{E} \mathcal{N} \mathcal{G}_{\text {kin }}\right)$, potential $\left(\delta \mathcal{E} \mathcal{N} \mathcal{G}_{\text {pot }}\right)$ and internal energy $\left(\delta \mathcal{E} \mathcal{N} \mathcal{G}_{\text {int }}\right)$. The grid point energy $(\mathcal{E N G})$ is obtained from the integration of the volumetric density of energy $(\delta \mathcal{E N G})$ across the volume of the grid point $(\delta \mathcal{V}$, see Eq. 1$)$

$$
\begin{aligned}
\mathcal{E N G} & =\delta \mathcal{E N} \mathcal{G}_{\text {kin }} \delta \mathcal{V}+\delta \mathcal{E N} \mathcal{G}_{\text {pot }} \delta \mathcal{V}+\delta \mathcal{E} \mathcal{N} \mathcal{G}_{\text {int }} \delta \mathcal{V} \\
\delta \mathcal{E N} \mathcal{G}_{\text {kin }} & =\frac{1}{2}\|\boldsymbol{v}\|^{2} \rho=\frac{1}{2}\left(u^{2}+v^{2}\right) \rho \\
\delta \mathcal{E N} \mathcal{G}_{\text {pot }} & =g H \rho \\
\delta \mathcal{E N} \mathcal{G}_{\text {int }} & =C_{v} T \rho \\
\delta \mathcal{V} & =\frac{d s^{2} \Delta p}{\rho g}
\end{aligned}
$$

where $\|\boldsymbol{v}\|$, wind speed; $\rho$, density; $g$, gravity; $H$, geopotential height; $C_{v}$, heat capacity at constant volume; $T$, temperature; $d s^{2}$, areal size of the grid point; $\Delta p$, pressure variation between bottom and top of the grid cell.

PV inverted fields used to modify the initial conditions imply a change in the total amount of energy in the atmospheric domain of the simulation. In this study that change will be computed as the mean absolute variation of the total energy $(\mathcal{M} \mathcal{A} \mathcal{V}$, Eq. 2)

$\mathcal{M} \mathcal{A} \mathcal{V}_{\chi}=\frac{\sum_{i, j, k}^{N i, N j, N k}\left|\chi_{\bmod }(i, j, k)-\chi_{\mathrm{ref}}(i, j, k)\right|}{N i \times N j \times N k}$,

where $\chi_{\bmod }(i, j, k)$ is the modified energy at each grid point; $\chi_{\text {ref }}(i, j, k)$ the unperturbed field and $N_{i}, N_{j}, N_{k}$, the number of grid points in each direction.

The proposed normalisation of the perturbations is realised by imposing the same energy variation in the initial conditions for each anomaly. Although each PV anomaly will be modified with a different pattern, the environment and the total amount of introduced/removed energy is forced to be the same. This condition will be condensed on a given percentage of the inverted balanced fields to be used to modify the initial conditions.

In the November 2001 case, the Upper level disturbances (ErPVp01 and ErPvpHigh) are clearly stronger than the surface thermal one (ErPVpTerm in Fig. 6). Therefore, the surface thermal anomaly will be used as a reference. Thus, $70 \%$ of the PV inverted fields from the surface thermal anomaly will determine a fixed energy perturbation of about $0.145 \mathrm{PJ}$ $\left(\mathrm{PJ}=10^{15} \mathrm{~J}\right)$ on the initial conditions. This energy value is used to fix the perturbations when the upper level anomalies are used. A Newtonian or Bisection iterative numerical 
method (Arfken, 1985) is used. This method is used to obtain the percentage of the inverted fields from ErPVp01 and ErPVpHigh that preserves the same amount of energy variation as the ErPVpTerm modification. The results of this numerical computation are summarised in Table 1.

Different perturbation energy profiles are derived from the anomalies, as it is shown in Fig. 7. ErPVpTerm influences much more the low levels, whereas ErPVp01 and ErPVpHigh influence strongly the upper levels. ErPVp01 is more evenly distributed in the vertical than ErPVpHigh, depicting the structural differences between troughs or cutoff lows and blocking anticyclones (Hoskins et al., 1985; Thorpe, 1986).

Figure 8 gives spatial information on the impact of each anomaly over the initial conditions. It shows the threedimensional effect on the total energy. ErPVpTerm extracts and adds energy to the environment. Since ErPVpTerm anomaly is aimed at capturing the surface baroclinicity of the initial environment, it has been constructed with both the positive and negative surface thermal anomaly. Perturbations carried out with a positive(negative) thermal anomaly is related to a decrease(increase) in the total energy. Upper level positive(negative) potential vorticity perturbations are related to a decrease(increase) in the total energy (see Fig. 9). Results of ErPVpTerm anomaly (top panel in Fig. 9) do not show an upward decrease profile as the $\mathcal{M A V}$ value (dash and cross line in Fig. 7). This is because the ErPVpTerm is based on both positive and negative thermal anomalies (top Fig. 6). As a result, the inverted fields increase/decrease the energy of the environment. Figure 9 gives the averaged total variations of this energy (thus allowing compensation between terms); meanwhile, in the $\mathcal{M A V}$ computation this is not allowed, since absolute values are used.

The perturbation energy partition among kinetic, potential and internal for each anomaly is shown in Fig. 9. ErPVpTerm is related to a variation in the internal and potential energies. The effect is almost constant at all vertical levels. ErPVp01 and ErPVpHigh energy contributions are broadly similar. These anomalies have an important effect on the potential and internal energy, with the maximum energy variation found at upper levels. Significant perturbations of the kinetic energy are also found at upper levels, where the anomalies are defined. ErPVp01 has the highest impact on the potential energy, while ErPVpHigh has the highest impact on the internal energy.

However, one cannot infer directly that an increase or decrease in the available energy to the environment will produce deeper or weaker systems. As it has been shown, increasing the upper level positive PV anomaly induces a significant decrease in the potential and internal energy, despite the fact that a strong upper level positive PV anomaly is usually related to strong cyclogenesis (Pettersen and Smebye, 1971; Hoskins et al., 1985). Energy impacts are shown as horizontally averaged values. Any information about changes in morphology and position of the features in the

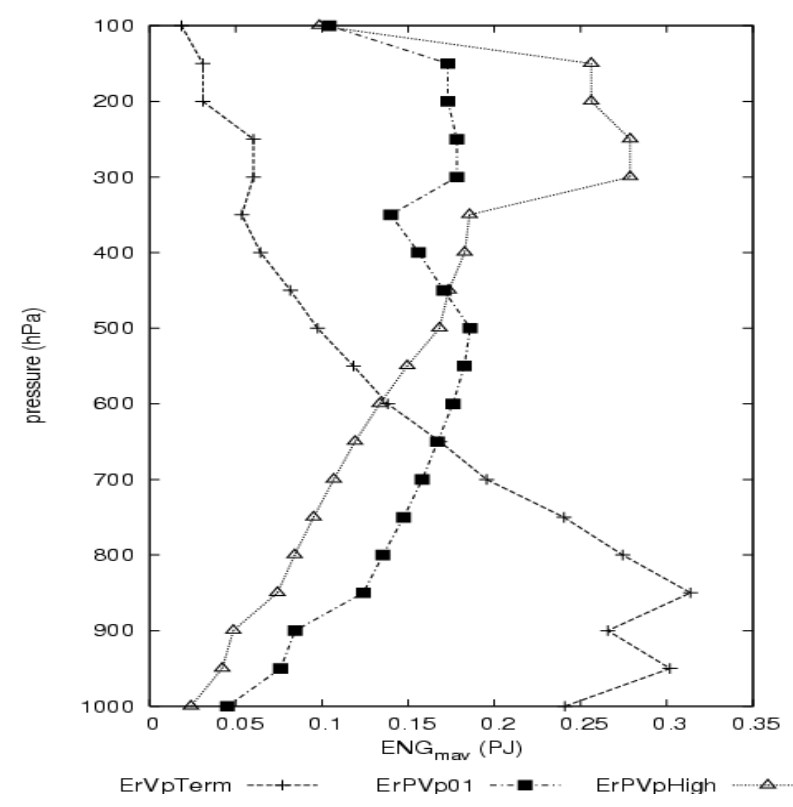

Fig. 7. Vertical profile of the mean absolute energy variation $(\mathcal{M} \mathcal{V})$ of the initial conditions according to the positive percentage found for each anomaly. ErPVpTerm (cross), ErPVp01 (filled square), ErPVpHigh (triangles). Note that the same area is enclosed by each curve (same total energy).

fields, from which genesis and maintenance of the atmospheric systems are explained, is not provided. Energetical implications for the baroclinic instability (Robinson, 1989) is out of scope of this study.

\section{Results of the simulations}

The sensitivity to changes in the initial conditions will be derived from the differences between the collection of simulated cyclones. The impacts on the simulated trajectories, central pressure value and central vorticity of the cyclones will be examined. Besides, a short description of the upper level vortex $(\mathrm{C} 0)$ properties and evolutions will be given. The computation of the vorticity characteristics of the cyclones follow the criteria of Campins et al. (2000), where a $200 \mathrm{~km}$ gridlength mesh is used.

Generally, simulated cyclones show a symmetric response between positive and negative modifications of the initial fields. That is, the two cyclones show the same variations, with opposite sign, from the control one (not shown). These differences are not very strong, owing to the small degree of modification introduced.

\subsection{Effects of the ErPVpTerm perturbation}

The modification of the environmental baroclinicity $( \pm$ ErPVpTerm) reveals significant variations with respect to the 

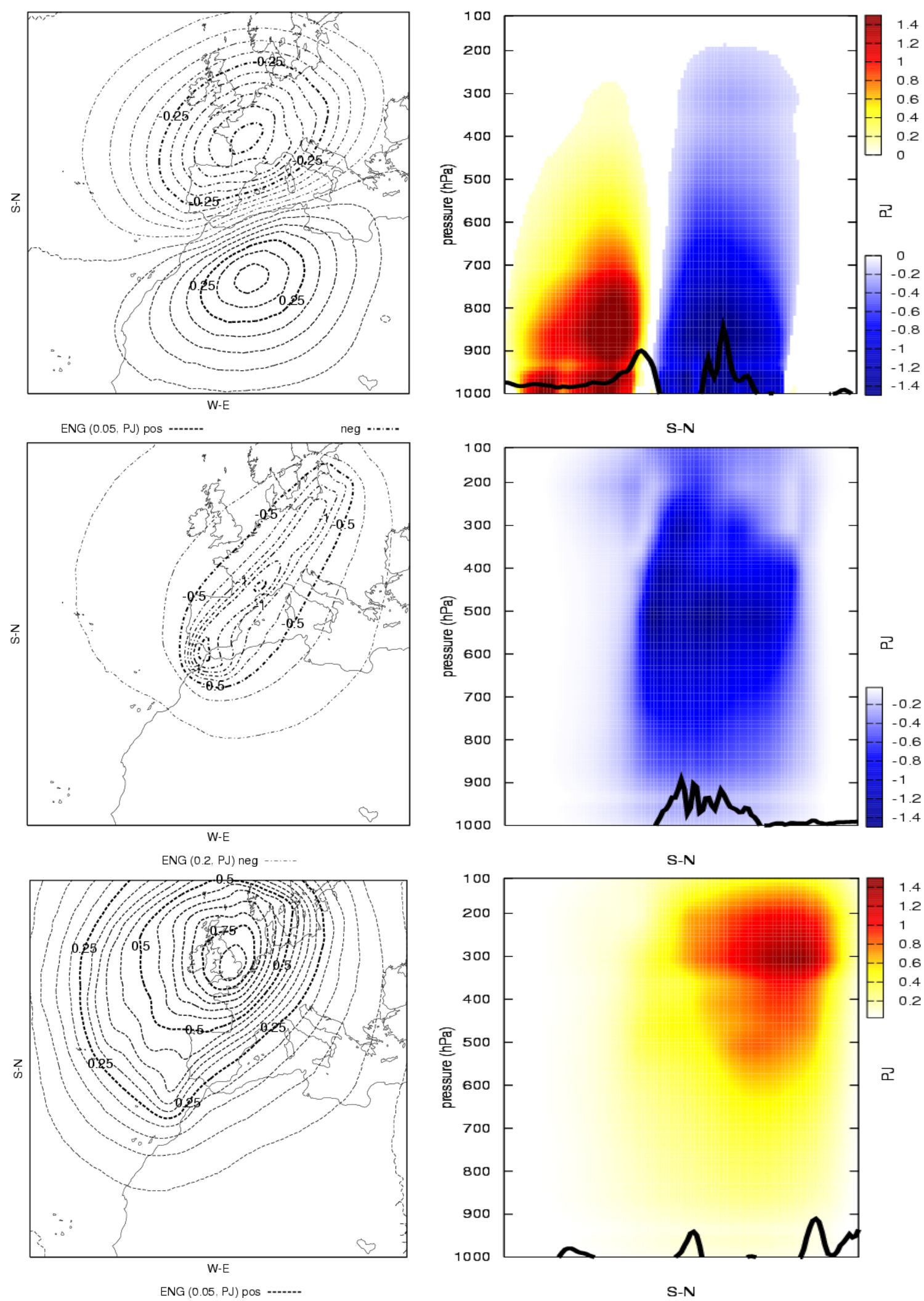

Fig. 8. Energy variations (PJ) introduced after the modification of the initial conditions (10 November 2001 at 00:00 UTC), according to each positive percentage of modification of the PV Inverted fields of each anomaly; ErPVpTerm (top), ErPVp01 (middle), ErPVpHigh (bottom). At $500 \mathrm{hPa}$ (left) and following vertical cross sections defined at Fig. 6 (right). 

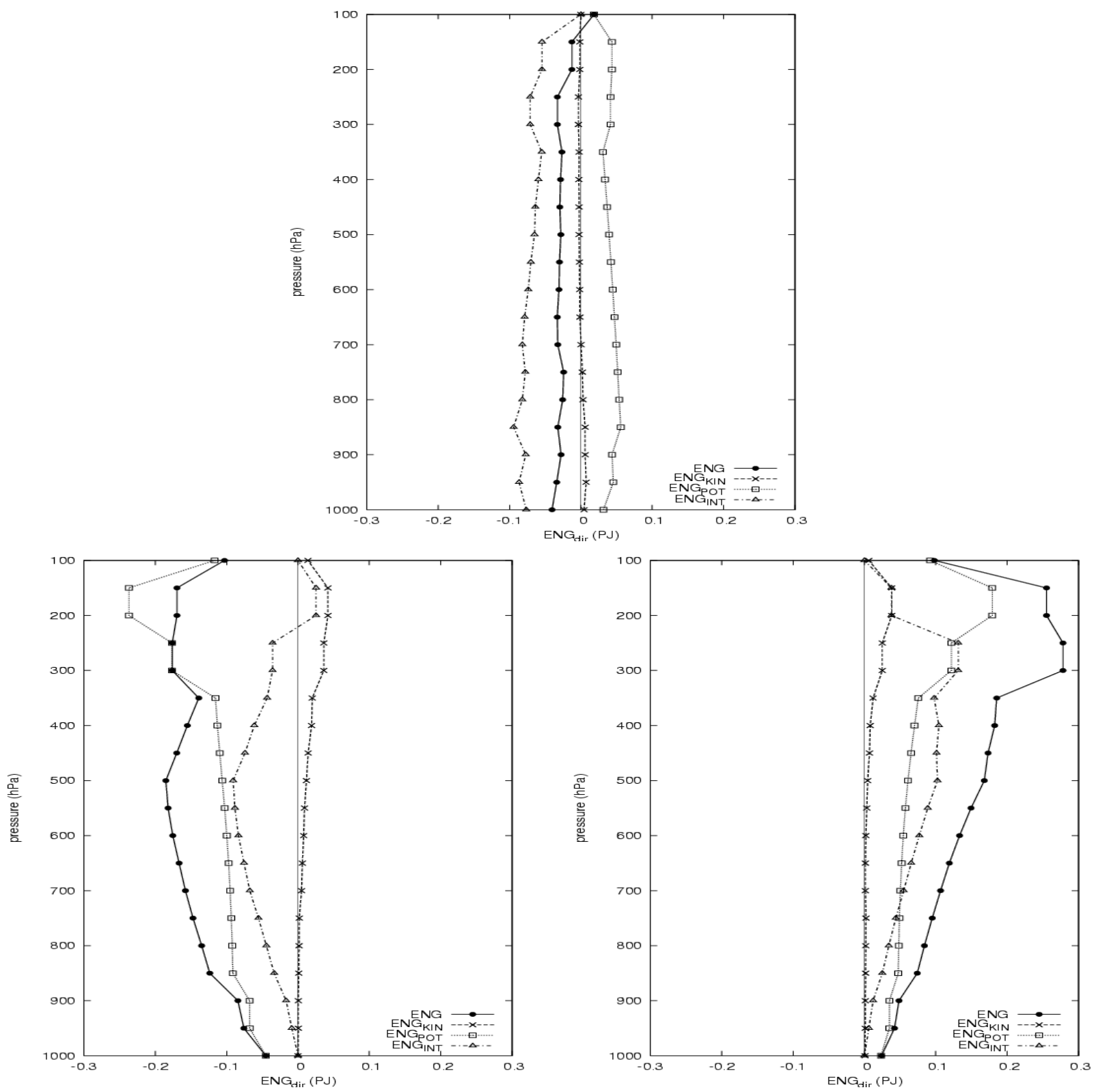

Fig. 9. Vertical profiles of the horizontally averaged total variations of energy (in PJ) according to the positive percentage of modification for each anomaly. Total energy variation (solid line, filled circles), Potential energy $\left(\mathcal{E} \mathcal{N} \mathcal{G}_{\text {pot }}\right.$, dotted line with squares), Kinetic energy $\left(\mathcal{E N} \mathcal{G}_{\text {in }}\right.$, dashed line with crosses), Internal energy $\left(\mathcal{E N} \mathcal{G}_{\text {int }}\right.$, dot-dash line with triangles). ErPVpTerm (top), ErPVp01 (bottom left), ErPVpHigh (bottom right).

control simulation during the initial and last phases of the cyclone evolution. These differences are the largest ones among the entire set of perturbations at the initial phase of the simulations. According to the results, the differences in the initial position of the cyclone are about $480 \mathrm{~km}$ (see Fig. 11), and for the initial central pressure value of the cyclone approx. $\pm 4 \mathrm{hPa}$ (see Fig. 12). At the same time, the simulated cyclones show strong variations in their vorticity (about $\pm 5 \mathrm{~s}^{-1}$ at $200 \mathrm{~km}$ gridmesh point, in Fig. 14). As a result of the change in the initial position of the cyclone, the interaction between the upper and low level disturbances has also changed (the relative phase between the disturbances is significantly changed in comparison to the other simulations, Fig. 13).

In the -ErPVpTerm(+ErPVpTerm) case (perturbation of the initial conditions by subtracting(increasing) the fields related to the horizontal thermal anomaly) the initial trajectory of the cyclone is clearly different from the control one (see 

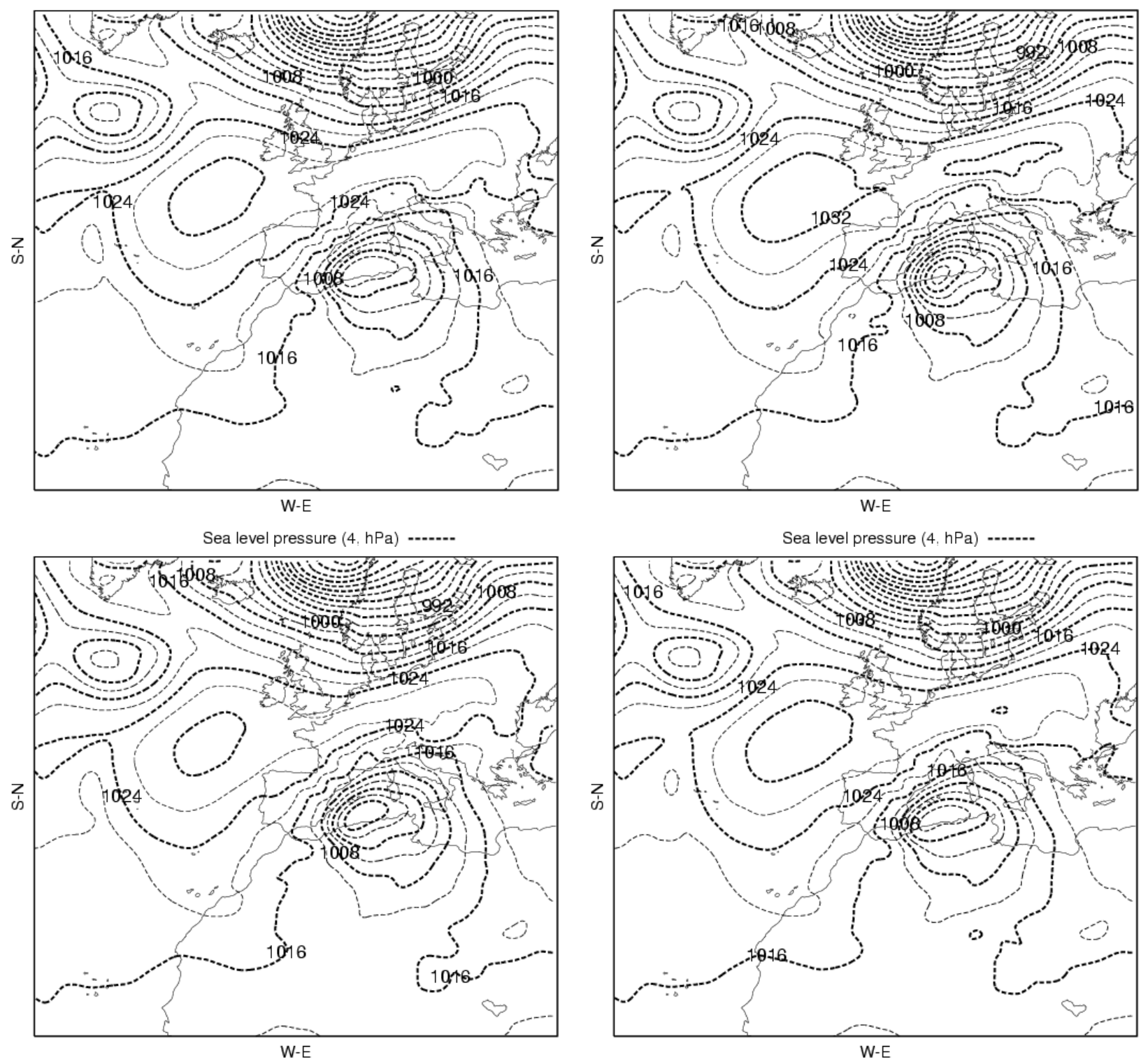

Sea level pressure $(4, \mathrm{hPa})$

Sea level pressure $(4, \mathrm{hPa})$----.--

Fig. 10. Sea level pressure at the mature state of the cyclone (11 November 00:00 UTC). Lines every $2 \mathrm{hPa}$. For the control simulation (top left), +ErPVpTerm (top right), +ErPVp01 (bottom left) and +ErPVpHigh (bottom right).

the two dashed lines with crosses in Fig. 11). The mature cyclones at 11 November 00:00 UTC show the largest variations with the control one: on the lowest central pressure value ( $\pm 6 \mathrm{hPa}$, Fig. 12), the relative distance with $\mathrm{C} 0$ (between $100-200 \mathrm{~km}$ in Fig. 13) and the central value of the upper level trough $\mathrm{C} 0$ about 1 with the PVU less than in the control one, (Fig. 13). ErPVpTerm perturbations have a deep impact when the cyclone crosses the Atlas mountains, modifying the relation between the cyclone and the upper level trough. This is shown in the phase-relativedistance (Fig. 13). As result, the perturbed cyclone from
-ErPVpTerm changes to a negative relative phase ( $\mathrm{C} 0$ is located in an eastern position relative to the cyclone) $9 \mathrm{~h}$ later than the + ErPVpTerm cyclone.

Initially, the $\mathrm{C} 0$ simulated from the \pm ErPVpTerm perturbations is similar with respect to the control one. However, at the mature state of the cyclone, $\mathrm{C} 0$ values are about $1 \mathrm{PVU}$ lower (see Fig. 13). The strong differences may be related to the change in the phase of the mutual interaction with the surface cyclone, attributed to the baroclinic deepening processes. This aspect is also reflected in the +ErPVpTerm cyclone simulation. The cyclone exhibits a stronger vorticity 

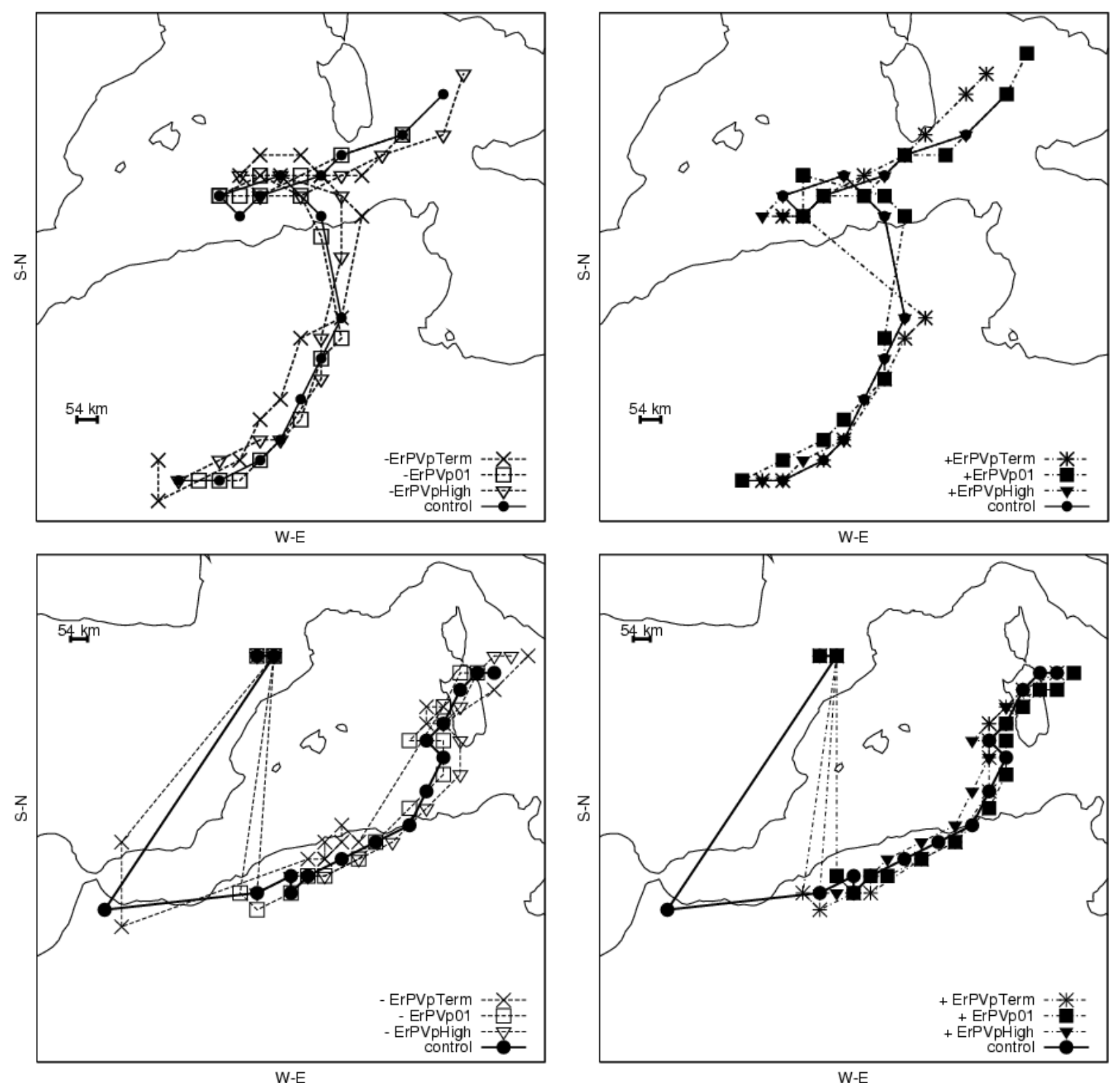

Fig. 11. Top panels: Trajectories of the cyclone obtained from the initially perturbed simulations. Bottom panels: Trajectory of C0 for the different simulations. For the negative modifications (left), for the positive modifications (right). According to values on Table 1. - ErPVpTerm (simple cross), +ErPVpTerm (full cross), -ErPVp01 (empty square), +ErPVp01 (filled square), -ErPVpHigh (empty triangle), +ErPVpHigh (filled triangle).

than the control one (see Fig. 14), associated with a deeper cyclone and a stronger North Atlantic high, which results in much stronger surface pressure gradients (see Fig. 10). During the dissipative phase of the cyclone (from 11 November at 12:00 UTC to 12 November at 00:00 UTC), the differences regarding the control cyclone become strong. The final position is clearly shifted about $54 \mathrm{~km}$ from the control one in both cases (Fig. 11), the central pressure value is changed by about $\pm 2 \mathrm{hPa}$ (Fig. 12), and the final values of $\mathrm{C} 0$ are stronger (about $\pm 1.5 \mathrm{PVU}$, Fig. 13). At the end of the simulated period a lower vorticity of the -ErPVpTerm cyclone is obtained due to the smaller dimensions of the cyclone, in comparison to the +ErPVpTerm one (not shown). The strongest deepening of the cyclone occurred between 10 November at 18:00 UTC and 11 November at 06:00 UTC (see Fig. 12). In contrast with the strong sensitivity to the ErPVpTerm anomaly, the growth rate of the cyclone does not vary significantly (about $-8 \mathrm{hPa} / 12 \mathrm{~h}$ in positive case and $-6 \mathrm{hPa} / 12 \mathrm{~h}$ in the negative). 

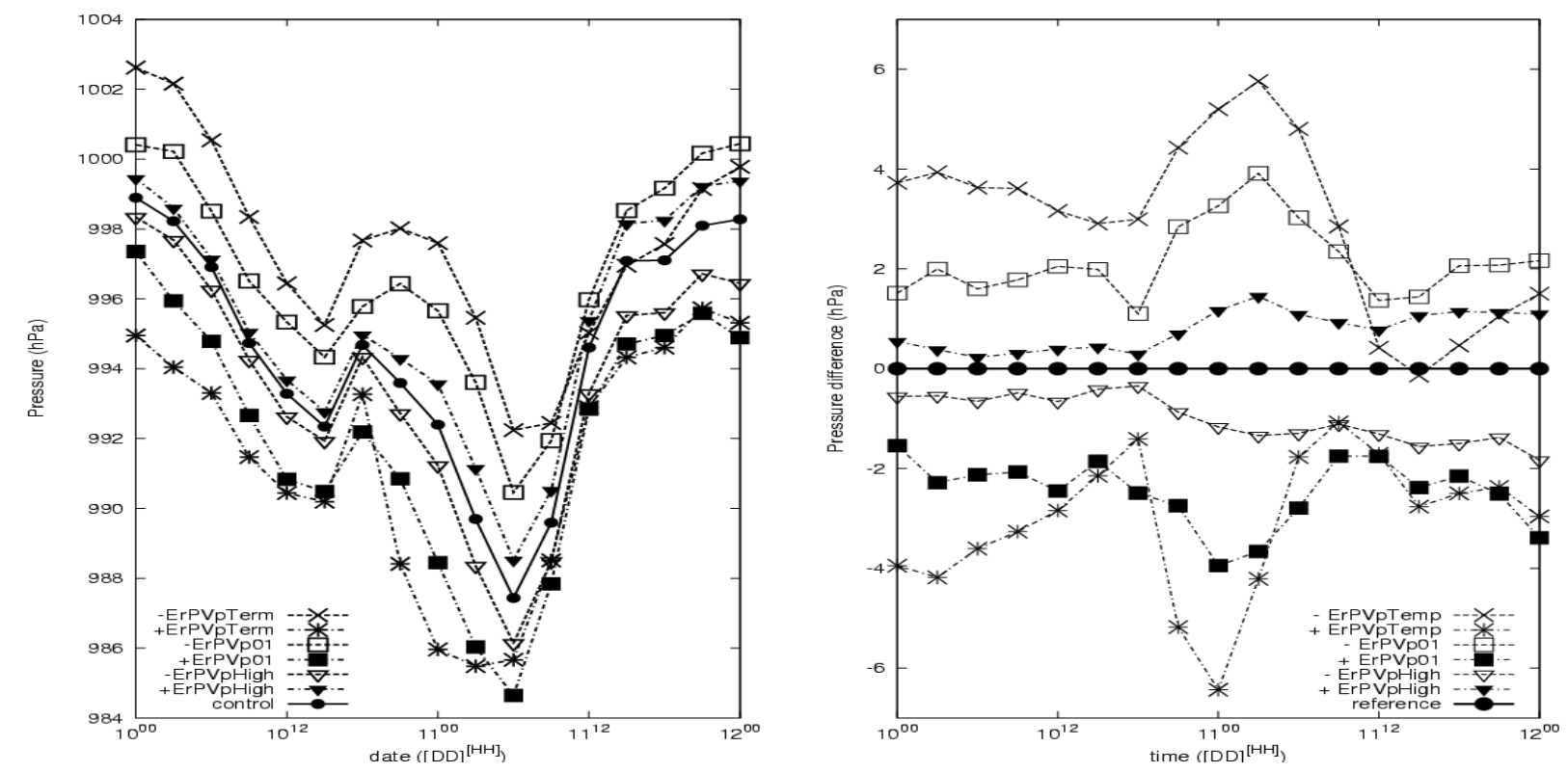

Fig. 12. Labels as in Fig. 11, but left panel: evolution of the central pressure of the cyclone. Right panel: evolution of the central pressure of the cyclone rescaled to control simulation results.
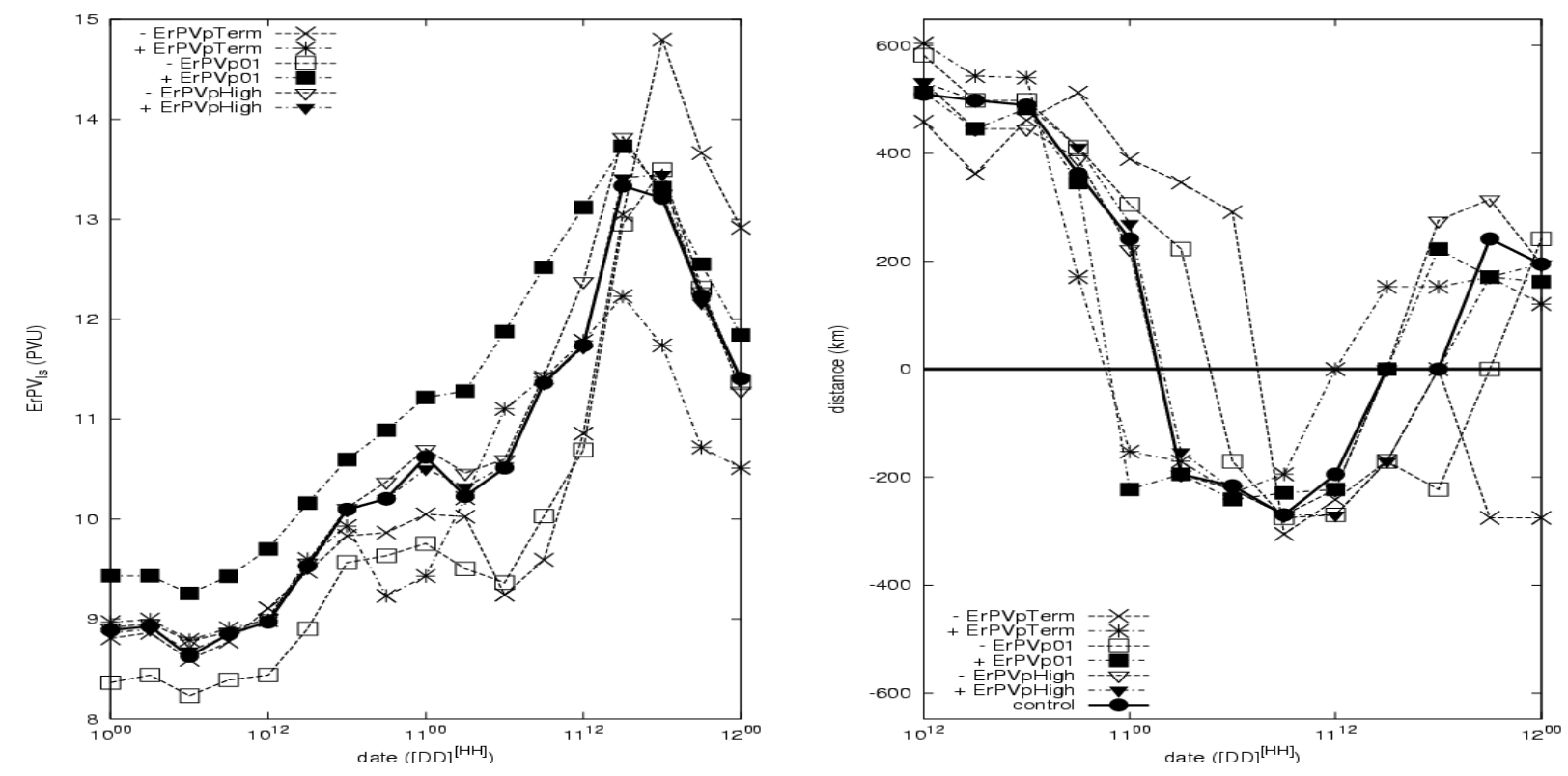

Fig. 13. Labels as in Fig. 11, but left panel: evolution of the C0 central value (PVU). Right panel: Evolution of the relative distance (km) between $\mathrm{C} 0$ and cyclone, positive(negative) sign denotes eastward(westward) relative position of the cyclone (starting date on 10 November at 12:00 UTC).

\subsection{Effects of the ErPVp01 perturbation}

The cyclone simulated through the \pm ErPVp01 perturbations reveals significant impacts on the upper level trough. The effects are most notable during the mature state of the cy- clone, on 11 November at 00:00 UTC. The simulated cyclones and the $\mathrm{C} 0$ centres describe similar trajectories as the control ones (see lines with squares in Fig. 11). However, the evolution of the central surface pressure values and the $\mathrm{C} 0$ magnitudes have been changed (see lines with squares in 

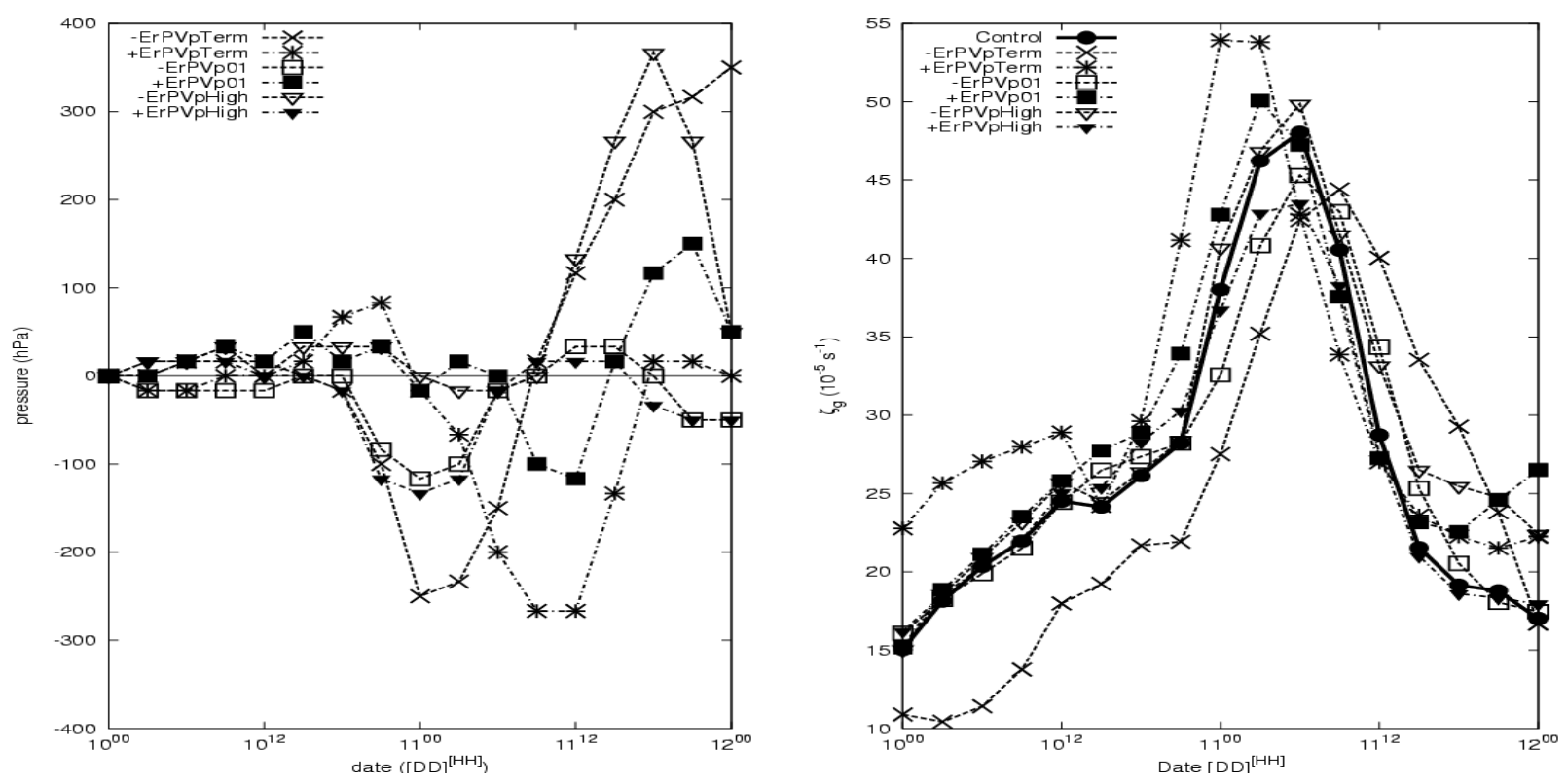

Fig. 14. Labels as in Fig. 11, but left panel: evolution of the depth of the cyclone rescaled to control simulation results (defined as the first minimal vorticity value above the centre of the cyclone, left). Right panel: evolution of the vorticity of the cyclone at a distance of $200 \mathrm{~km}$ (defined like in Campins et al., 2000). Each figure has been temporally filtered through a mobile-average filter of 3 time-points.

Figs. 12, 13). Low differences are observed between the final PV values of $\mathrm{C} 0$, between the +ErPVp01 and -ErPVp01 cases (Fig. 13), probably due to diabatic influences.

Initially, the perturbation of ErPVp01 is localized at upper levels, where the trough is altered (central pressure of the cyclones for $\pm \mathrm{ErPV} 01$ cases are initially only $\pm 1 \mathrm{hPa}$ different than the control one). During the initial phase, the cyclones preserve similar vorticities (see Fig. 14), but they change significantly their dimensions (not shown). A stronger or weaker upper level PV vortex does not produce any strong effect on the trajectory of the disturbances (similar trajectories as the control one are obtained; see Fig. 13). However, a weaker upper level disturbance ( - ErPVp01 case) decreases the upper-low level interaction and this is depicted as a shallower mature cyclone. With a stronger upper level trough (+ErPVp01 perturbation) the cyclone moves faster than the control one (Fig. 11). Significant geostrophic vorticity differences are obtained at the mature stage of the cyclone (11 November at 00:00 UTC, Fig. 14), as a reflection of the changes in the vorticity advections due to a stronger(weaker) upper level trough. In the last phase of the evolution of the cyclone, the $\mathrm{C} 0$ value is similar to the control one, but central pressure values of the cyclone are clearly different (about $\pm 2 \mathrm{hPa}$ ), possibly related to changes in the relative phase between $\mathrm{C} 0$ and the cyclone at this ending phase. As for the ErPVpTerm anomaly simulations, small differences between the positive/negative cases in the maximum growth rate of the cyclone are also observed for this anomaly.

\subsection{Effects of the ErPVpHigh perturbation}

The cyclones obtained in the simulations with the ErPVpHigh anomaly present the lowest sensitivities. The differences with respect to the control simulation are rather low until the last phase of the cyclone. The largest variations are obtained in the -ErPVpHigh case. A weaker North Atlantic anticyclone (as a result of the -ErPvpHigh initial perturbation) can help create a faster movement of the Mediterranean disturbance, owing to a weaker blocking of the anticyclone. Final values of the $\mathrm{C} 0$ centre and the relative distances between the low and upper level disturbances do not differ from the control one (see Fig. 13), but final phase trajectories are significantly shifted from the control one (see lines with triangles in Fig. 11).

\section{Conclusions}

Piecewise PV Inversion techniques, combined with perturbed numerical simulations, have been used as a tool to dynamically study various features of atmospherics systems. These techniques can offer useful information about mechanisms and roles of a wide range of features involved in the lifecycle of the disturbances. Although numerical solutions to well-defined inversion equations are determined, some uncertainties and subjectivities arise in its application. Some of these case-dependent aspects are: computation of a reference state from which to define the PV anomalies (zonal mean, temporal mean, number of members to establish an 
average, etc.), morphology and magnitude of the anomalies, and degree of modification of the initial conditions. In the present study an objective procedure has been proposed as a method to quantify the latter aspect of the technique. In this way, one can diminish the ambiguity on the use of the PV Inversion technique applied to modify the initial conditions of numerical simulations.

It is proposed to use the total Mean Absolute Variation $(\mathcal{M A V})$ of energy introduced, due to the modification of the initial conditions. The Root Mean Square Variation $(\mathcal{R M S} \mathcal{V})$ could have been proposed, but by squaring the values, contributions of big and small energetic variation values are differently, weighted in the $\mathcal{R M S V}$ calculation. The partition of the energy into mechanical, internal and kinetical energies can be useful information from which a deeper understanding of the role of the collection of features can be obtained.

The 9-12 November 2001 Mediterranean cyclone has been studied in terms of its sensitivity to changes in the initial conditions due to three PV anomalies: the surface thermal anomaly related to the origin of the cyclone and the baroclinic initial conditions of the environment; the upper level trough related to the baroclinic growth mechanism of the cyclone; the upper level North Atlantic high pressure zone related to the environmental conditions of the upper troposphere. The anomalies show different morphologies and intensities, but since the energy-quantification of the initial modification has been applied, sensitivity results might be only attributed to the dynamical role of the anomalies. The surface thermal anomaly is revealed as the most important feature of the initial conditions for the cyclogenesis. A significant effect on the initial central pressure of the cyclone and a weak effect on its initial position are shown. Both have a strong effect on the resultant evolution of the case. The sensitivities to changes in the initial conditions due to the upper level disturbances are also important. Nevertheless, the initial impact on the structure of the cyclone is lower than for the thermal anomaly, but in later stages of the episode the impact becomes the strongest. The effects of changes in the initial conditions associated with the North Atlantic high are weak. However, in the last phase of the cyclone evolution, the initial perturbations show a notorious impact on the trajectory of the cyclone.

The sensitivity results are strongly affected by the baroclinic mechanism. It is illustrated that the diversity of simulated cyclones can be mainly explained as a function of the changes in the vertical tilt between the upper level trough and the surface disturbance. The sensitivity test to the increase(decrease) in the surface thermal anomaly shows that with similar values of the upper level disturbance, changes in the relative position and central pressure value of the surface disturbance generates deeper(weaker) and faster(slower) evolution of the simulated cyclone. This case also emphasises the mutual interaction between anomalies, in the sense that the thermal anomaly perturbation does not affect the ini- tial structure and intensity of the upper level disturbance, but during the simulated period, the upper level disturbance strength clearly differs between both experiments. The upper level disturbance perturbations modify both the initial central value of the cyclone and the upper level trough, but only slightly modify the relative distance between both features. These variations generate a strongly varied evolution of the cyclone. Finally, the North Atlantic high does not exhibit a significant role on the upper level disturbance, on the surface cyclone strength or on the relative position between disturbances. However, the small perturbations on the relative position between the upper and low level disturbances seem to be enough to originate significantly different final cyclones.

Finally, the proposed quantification method of the piecewise PV inversion derived perturbations can be applied as a general methodology in dynamic meteorology. The application to various events would allow an objective intercomparison between cases, independent of the morphology, characteristics and origin of the selected anomalies. This method of quantification could contribute to the PV study and analysis based on the most important features involved in the evolution of the cyclones or other atmospheric phenomena.

Acknowledgements. Support from MEDEXIB (REN 2002-03482) and PRECIOSO (CGL2005-03918/CLI) projects and PhD grant BES-2003-0696 (all from the Spanish "Ministerio de Educación y Ciencia") are acknowledged. V. Homar for the discussions and commentaries is also acknowledged. J. Campins for the commentaries and help. Á. Luque for the satellite image.

Topical Editor U.-P. Hoppe thanks two anonymous referees for their help in evaluating this paper.

\section{References}

Arfken, G.: Mathematical Methods for Physicists, 3rd ed., Orlando, FL: Academic Press, 1985.

Argence, S., Lambert, D., Richard, E., Söhne, N., Chaboureau, J.P., Crépin, F., and Arbogast, P.: High resolution numerical study of the Algiers 2001 flash flood: sensitivity to the upper-level potential vorticity anomaly, Adv. Geosci., 7, 251-257, 2006, http://www.adv-geosci.net/7/251/2006/.

Bleck, R.: Depiction of upper/lower vortex interaction associated with extratropical cyclogenesis, Mon. Weather Rev., 118, 573585, 1990.

Bluestein, H. B.: Synoptic-Dynamic Meteorology in Midlatitudes. Volume 1, Oxford university press, Inc., 1992.

Bretherton, F. P.: Critical layer instability in baroclinic flows, Q. J. Roy. Meteor. Soc., 92, 325-334, 1966.

Campins, J., Genovés, A., Jansà, A., Guijarro, J. A., and Ramis, C.: A catalogue and a classification of surface cyclones for the western Mediterranean, Int. J. Climatol., 20, 969-984, 2000.

Campins, J., Jansà, A., and Genovés, A.: Three-dimensional structure of western Mediterranean cyclones, Int. J. Climatol., 26, 323-343, 2006.

Charney, J. G.: The use of primitive equations of motion in numerical prediction, Tellus, 7, 22-26, 1955. 
Davis, C. A. and Emanuel, K. A.: Potential vorticity diagnostics of cyclogenesis, Mon. Weather Rev., 119, 1929-1953, 1991.

Davolio, S. and Buzzi, A.: A nudging scheme for the assimilation of precipitation data into a mesoscale model, Weather Forecast., 19, 855-871, 2004.

Grell, G., Dudhia, J., and Stauffer, D.: A description of the fifthgeneration Penn State/NCAR mesoscale model (MM5), NCAR Technical Note, NCAR/TN-398+STR, 117pp, 1994.

Gyakum, J. R.: On the Evolution of the $Q E$ II Storm. II: Dynamic and Thermodynamic Structure, Mon. Weather Rev., 111, 1156$1173,1983 b$.

Hakim, G. J., Keyser, D., and Bosart, L. F.: The Ohio valley wavemerger cyclogenesis event of 25-26 january 1978. Part II: Diagnosis using quasigeostrophic potential vorticity inversion, Mon. Weather Rev., 124, 2176-2205, 1996.

Homar, V., Ramis, C., and Alonso, S.: A deep cyclone of African origin over the Western Mediterranean: diagnosis and numerical simulation, Ann. Geophys., 20, 93-106, 2002, http://www.ann-geophys.net/20/93/2002/.

Homar, V., Romero, R., Stensrud, D., Ramis, C., and Alonso, S.: Numerical diagnosis of a small, quasi-tropical cyclone over the western Mediterranean: Dynamical vs. boundary factors, Q. J. Roy. Meteor. Soc., 129, 1469-1490, 2003.

Horvath, K., Fita, L., Romero, R., and Ivancan-Picek, B.: A numerical study on the first phase of a deep Mediterranean cyclone: Cyclogenesis in the lee of the Atlas Mountains, Meteorologische Z. 1, 15, 133-146, 2006.

Hoskins, B. J., McIntyre, M. E., and Robertson, A. W.: On the use and significance of isentropic potential vorticity maps, Q. J. Roy. Meteor. Soc., 111, 877-946, 1985.

Huo, Z., Zhang, D.-L., Gyakum, J. R., and Stainforth, A. N.: A diagnostic analysis of the superstorm of march 1993, Mon. Weather Rev., 123, 1740-1761, 1995.

Huo, Z., Zhang, D.-L., Gyakum, J. R., and Stainforth, A. N.: Interaction of potential vorticity anomalies in extratropical cyclogenesis. Part I: Static Picewise Inversion, Mon. Weather Rev., 127, 2546-2561, 1999a.
Huo, Z., Zhang, D.-L., Gyakum, J. R., and Stainforth, A. N.: Interaction of potential vorticity anomalies in extratropical cyclogenesis. Part II: Sensitivity to initial perturbations, Mon. Weather Rev., 127, 2563-2575, 1999b.

Kuo, Y. A., Shapiro, M. A., and Donall, E. G.: The interaction between baroclinic and diabatic processes in a numerical simulation of a rapidly intensifying extratropical marine cyclone, Mon. Weather Rev., 119, 368-384, 1991a.

Kuo, Y.-H., Reed, R. J., and Low-Nam, S.: Effects of surface energy fluxes during the early development and rapid intensification stages of seven explosive cyclones in the western Atlantic, Mon. Weather Rev., 119, 457-476, $1991 b$.

Pettersen, S. and Smebye, S. J.: On the development of extratropical cyclones, Q. J. Roy. Meteor. Soc., 97, 457-482, 1971.

Reed, R. J., Stoelinga, M. T., and Kuo, Y.-H.: A model-aided study of the origin and evolution of the anomalously high potential vorticity in the inner region of a rapidly deeping marine cyclone, Mon. Weather Rev., 120, 893-913, 1992.

Robinson, W. A.: On the structure of potential vorticity in baroclinic instability, Tellus, 41A, 275-284, 1989.

Romero, R.: Sensitivity of a Heavy Rain producing Western Mediterranean cyclone to embedded Potential Vorticity anomalies, Q. J. Roy. Meteor. Soc., 127, 2559-2597, 2001a.

Sanders, F. and Gyakum, J. R.: Synoptic-Dynamic climatology of the 'Bomb', Mon. Weather Rev., 108, 1589-1606, 1980.

Thorpe, A. J.: Synoptic scale disturbances with circular symmetry, Mon. Weather Rev., 114, 1384-1389, 1986.

Tripoli, G. J., Medaglia, C. M., Dietrich, S., Mugnai, A., Panegrossi, G., Pinori, S., and Smith, E. A.: The 9-10 November 2001 Algerian Flood: A Numerical Study., B. Am. Meteorol. Soc., 86, 1229-1235, 2005. 